\title{
Numerical Framework to Determine Instability Modes for Static Vehicles under Partial Submergence
}

\author{
Syed Muzzamil Hussain Shah ${ }^{1}$, Zahiraniza Mustaffa ${ }^{1, *}$, Khamaruzaman Wan \\ Yusof $^{1}$
}

${ }^{1}$ Department of Civil and Environmental Engineering

Universiti Teknologi PETRONAS, 32610 Perak, MALAYSIA

*Corresponding Author

DOI: https://doi.org/10.30880/ijie.2019.11.01.020

Received 25 June 2018; Accepted 12 December 2018; Available online 15 May 2019

\begin{abstract}
Studies on instabilites of static (parked) vehicles that are partially submerged during flood events have captured worldwide attention in the previous years. The response of varying hydrodynamics effects to vehicles have been the interests reported. Indeed, the vehicle shapes have been found to significantly affect the response towards its hydrodynamics. These works, however, were experimental in nature. As the development of complex exterior designs of vehicles progressively made with the expansion of technologies, repeating the tedious experimental testing on another set of vehicle designs may not be practical. Herein this paper attempts to provide guidance on the use of a simple numerical framework to compensate the use of experimental testing. The proposed framework has been validated with a series of experimental testing conducted on a 1:24 scaled model that was controlled to be partially submerged under sub-critical conditions. Keeping close to the assumptions that the submergence levels of the vehicles are significantly influencing the buoyancy and drag forces, the numerical approach had given a simpler mechanism on the calculations of the submerged area $\left(A_{D}\right)$ and volume $(V)$ of the vehicle, when this could not be computed easily during experiments. The parameters $A_{D}$ and $V$ coupled with a graphical presentation of instability threshold values, would provide quick computations on the corresponding forces acting on the any similar characteristics static vehicle accordingly.
\end{abstract}

Keywords: Numerical approach, instability, static vehicle, partial submergence

\section{Introduction}

Rapid urbanization and land-use change are assumed to be significant factors in increasing disaster potential like floods, especially in rapidly expanding nations, of which Malaysia is an example [1]. Water is one of the most powerful forces on earth. It has the potential to cause major damages to anything that comes across it. People, vehicles and even buildings in the path of flowing tides can be vulnerable to being lifted, pushed and even washed away completely by the flood streams [2]. Concerning vehicles in floodwaters, its stability is jeopardized when the hydraulic variables, i.e, flow velocity (v) and water depth (y) exceeds certain limiting thresholds [3]. The loss of vehicle stability is not only limited to the hydraulic variables, but it also relies on the physical and geometric characteristics of the vehicle, its orientation towards the incoming flow direction and the degree of submergence [4].

The mechanisms that ultimately compromise the stability of a vehicle in floodwater are termed as sliding, floating and toppling motions. When a vehicle becomes unstable and loses its traction with road surface, sliding instability takes place. On the other hand, floating instability occurs when buoyancy and lift force exceeds vehicle weight [5]. A further mode of instability also known as toppling instability, appears to be restricted to vehicles which are already sliding or floating [6]. Therefore, in the current investigation, the first two modes of vehicle instability have been considered which are further illustrated in Figure 1. 


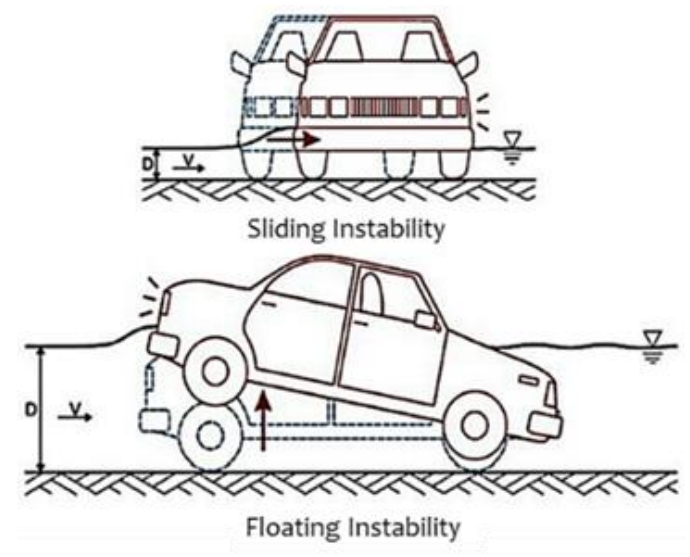

Fig. 1- Instability modes [5]

Earlier experimental investigations (1967-1973) on the stability of static vehicles (old-fashioned) were carried out by determining the vertical and lateral reactions on the vehicle recorded by the burette floats [6]. Later (2010-2017), the instability points for the static vehicles (modern) were measured by positioning the vehicles in the testing sections (hydraulic flume) on the desired slopes under varying flow rates and water depths until the movement of the vehicle was noticed. The measured flow velocity and water depth (v.y) values then defined one instability point in the velocitydepth domain [3]. Studies in the past have recognized that how the varying factor combinations of vehicle mass, dimensions, buoyancy and resistant forces could affect the vehicle stability in floodwaters. Concerning partial submergence, it has been identified that for every increment in the water depth, the amount of velocity needed to achieve vehicle instability is decreased. Simply put, the higher the water depth, lesser velocity is required to cause vehicle instability [7].

Herein an inventive approach has been proposed to estimate the influence of hydrodynamic forces on a flooded vehicle. Basically, the instability modes for a static vehicle are classified based on the dominancy of the hydrodynamic forces, namely buoyancy $\left(\mathrm{F}_{\mathrm{B}}\right)$, lift $\left(\mathrm{F}_{\mathrm{L}}\right)$, drag $\left(\mathrm{F}_{\mathrm{D}}\right)$ and frictional $\left(\mathrm{F}_{\mathrm{R}}\right)$ forces. However, due to small size and complex exterior design of the scaled models, the most challenging, by far, has been the estimation of vehicle's submerged volume $(\mathrm{V})$ and the area projected normal to the flow $\left(\mathrm{A}_{\mathrm{D}}\right)$. These parameters are essential for the theoretical assessment of buoyancy and drag forces. Herein these proportions have been estimated through a simple numerical framework which involves the use of Auto-Cad (2007), Solid-Works (2015) and ANSYS (Static Structural, Workbench 15.0). On the basis of the results obtained, it is believed that this simple approach offer possible ways to estimate the impact of various hydrodynamic forces at varying $(\mathrm{v} \cdot \mathrm{y})$ combinations.

\section{Theoretical Background}

The hydrodynamic forces are formed by moving water particularly floodwaters which are capable to drift vehicles on urban floodplains. Such flows usually exert the following forces, namely lift, drag and buoyancy force on a static vehicle. However, an additional force which resist the drag and allows the vehicle to remain stable on the ground is called frictional force [8]. Therefore, under such circumstances, vehicle stability is controlled by the above forces including the gravitational force as shown in Figure 2.

The buoyancy force which is the component of the vertical pushing force exerts pressure from the bottom on the immersed vehicle. Hence, the mechanism of floating instability takes place when this force together with the lift force exceeds vehicle weight. The buoyancy force can be given as:

$$
F_{B}=\rho g V
$$

where, $\rho$ is the water density, $\mathrm{g}$ is the acceleration due to gravity and $V$ is the submerged volume.

Irrespective of flow state, the impact of lift force has been completely disregarded and the impact of buoyancy force has been solely considered to cause floating instability [8]-[10]. Although, the impact of lift force is dominant for high flow velocities and under such circumstances the effect of buoyancy force could be neglected [3]. Herein the study was performed under sub-critical flow states where the impact of lift force was found insignificant. Thus, its impact on the static vehicle was disregarded and the impact of buoyancy force alone was considered to estimate floating instability failure. However, the lift force can be given as:

$$
F_{L}=\frac{1}{2} \rho C_{L} A_{L} v^{2}
$$


where, $C_{L}$ is the lift coefficient, $A_{L}$ is the vertically projected area of the vehicle which is equivalent to the crosssectional area of the vehicle base and $v$ is the velocity of the flow [3].

In fluid dynamics, drag acts opposite to the relative motion of any object moving with respect to a surrounding fluid. The drag force relies on the area of changing momentum, fluid velocity and its density. It can be expressed as:

$$
F_{D}=\frac{1}{2} \rho C_{D} A_{D} v^{2}
$$

where, $\rho$ is the density of water, $C_{D}$ is the drag coefficient, $A_{D}$ is the submerged area projected normal to the flow and $v$ is the velocity of the flow [8].

In context of vehicle stability in floodwaters, the friction force is the only force which prevents a static vehicle from sliding. This force opposes the drag caused by the incoming flow and acts between the floodplain surface and vehicle tires. It is proportional to the friction coefficient and the net weight of the vehicle which can also be given as:

$$
F_{R}=\mu F_{N}
$$

where, $\mu$ is the coefficient of friction, set at 0.3 after Bonham and Hattersley (1967) and $F_{N}$ is the normal reaction force from the ground which is equivalent to the net weight of the vehicle (axle load in dry conditions minus the buoyancy force) [10].

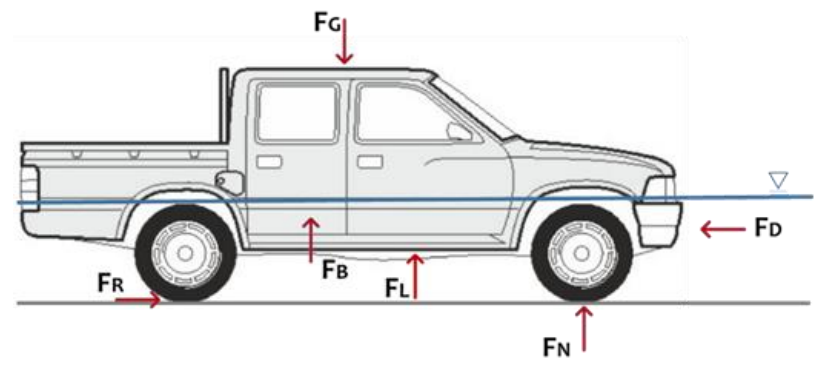

Fig. 2 - Forces acting on a static vehicle in floodwaters

\section{Theoretical Analysis}

Substantial changes have occurred in vehicle design, notably in vehicle aerodynamic shape, sealing capacity, ground clearance and weight, since those earlier investigations (1967-1993). Herein the study was performed on a modern vehicle, named Volkswagen Scirocco, which was scaled down to 1:24. The study was performed with the condition of rear tires being locked only. Further, to ensure proper sealing capacity, the interior space of the vehicle was filled with the light foam and to make it initially water tight, its side door glasses were covered with plastic. The specifications of the prototype and the model are shown in Figure 3 and 4, respectively. In the current study, more focus has been given to the numerical framework for the estimation of $A_{D}$ and $V$. Therefore, interested readers are recommended to refer to the experimental works of the authors on the instability thresholds, partial submergence, static vehicle and flat road surface for further clarification [11].

Herein the influence of hydrodynamic forces, namely frictional, buoyancy and drag forces on vehicle size, design shape and weight under partial submergence have been theoretically assessed. In the first section, the influence of vertical pushing force, namely buoyancy force on the mode of floating instability, whereas in the second section the impact of horizontal pushing force, namely drag and frictional forces on the mode of sliding instability have been discussed.

\subsection{Vertical Pushing Force}

To assess buoyancy force, the immersed volume of the vehicle $(V)$ at different depths, water density $(\rho)$ and acceleration due to gravity $(g)$ are required. The water density and acceleration due to gravity remains constant. Therefore, to estimate the up-thrust force, the immersed volume of the vehicle at different depths was needed. With that regards, the vehicle chassis was precisely structured to the given scale ratio through Auto-Cad (2007). A 2D design was initially prepared. The file was then saved as '*. $d w g$ ' format. It was then transferred to Solid-Works (2015) for further modification where the model was enhanced and extruded to prepare a complete 3D model of the chassis. Compared to the complex design of the chassis, the design of the ring-shaped tires was rather simplistic due to its simple geometry. However, the submerged volume of the chassis and the tires at different water depths was separately determined. Herein the study was performed on a flat road condition where the state of the flow remained sub-critical (behaved in a slow and stable way). Therefore, it was straight forward to estimate the submerged volume of the vehicle at the 
required water depths as it equally covered the vehicle chassis for every increment in depths. However, prior to crop the model at the desired height, the ground clearance of the vehicle was taken into consideration and it was ensured that the water depth was estimated exactly from the point where the tires were in contact to the surface. This file was then saved as '*.igs' format. Lastly, the saved file was then transferred to ANSYS (Static Structural, Workbench 15.0) and the submerged volume of the vehicle displaced by the water was obtained. Once the submerged volume of the chassis was known, it was then accumulated with the tires volume for the estimation of buoyancy force.
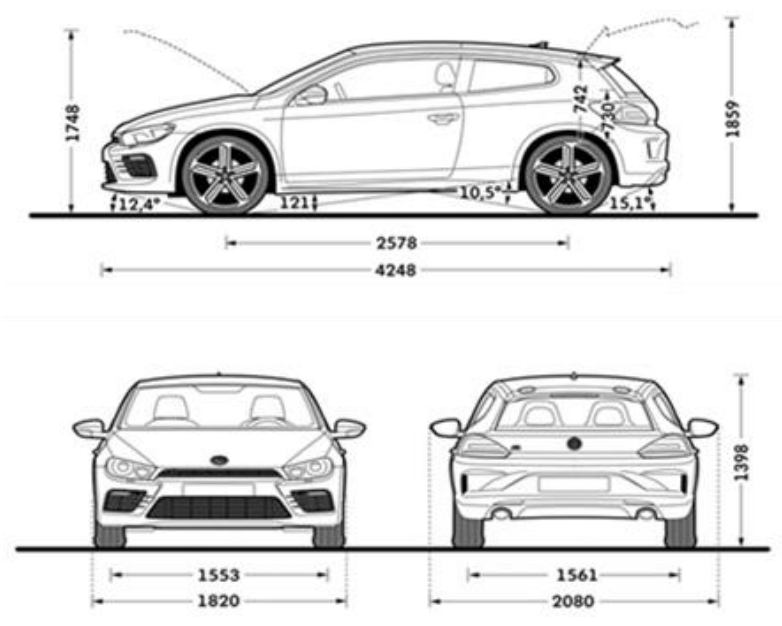

Fig. 3 - Exterior dimensions of the prototype, 1:1 (mm)

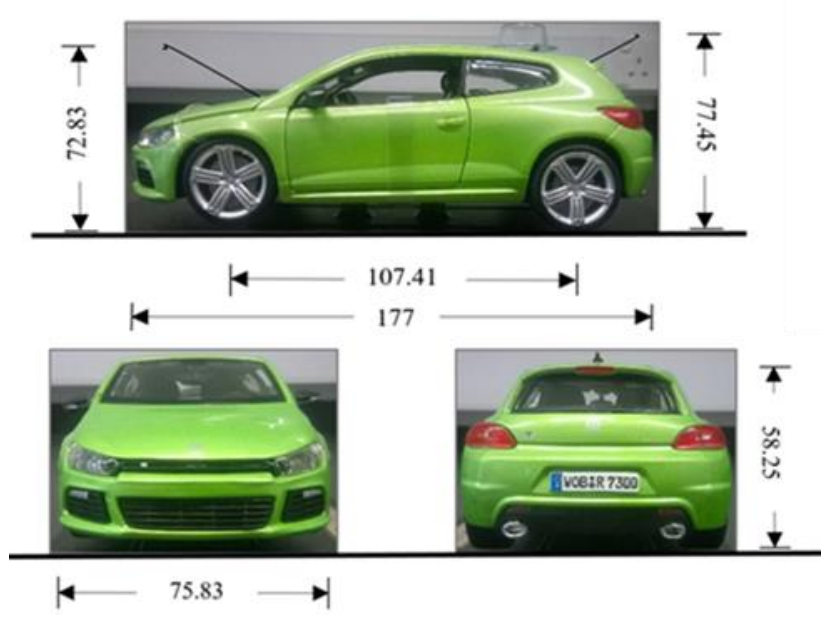

Fig. 4 - Exterior dimensions of the model, 1:24 (mm)

\subsection{Horizontal Pushing Force}

The drag force on the vehicle is usually induced by the approaching flow which if exceeds the resistive force between the ground surface and vehicle tires causes sliding instability. Herein the impact of drag force on the static vehicle at all orientations was noticed. Unlike buoyancy force, which relies on the submerged volume, the drag force depends on the flow velocity, drag coefficient and submerged area projected normal to the flow. Therefore, the influence of drag force varies with vehicle position and at varying $(\mathrm{v} \cdot \mathrm{y})$ combinations. However, for the estimation of drag force, water density $(\rho)$ was taken as $1000 \mathrm{~kg} / \mathrm{m}^{3}$, the drag coefficient $\left(C_{D}\right)$ was set to 1.1 or 1.15 depending on the depth of the floodwater level with respect to the vehicle chassis [12]. Further, to assess the submerged area $\left(A_{D}\right)$ of the vehicle, the submerged volume was fractionated by vehicle width for the given water depths. Lastly, the approaching flow velocity $(v)$ at different water depths was measured through velocity meter.

For the estimation of friction force, the net weight of the vehicle at different water depths and the friction coefficient between the ground surface and the tires are needed. Herein for every increment in the water depth, the net weight of the vehicle was obtained once the buoyancy force was known (axle load in dry conditions minus buoyancy force). Moreover, the friction coefficient was assumed to be 0.3 , which was confirmed almost adequate for the all surface types as proposed by Bonham and Hattersley [13]. 


\section{Numerical Framework}

In the given section, the detailed and step by step approach of drawing the 2D model on Auto-Cad (2007), the way how the model was extruded into a 3D model using Solid-Works (2015) and the estimation of submerged fractions of the vehicle through ANSYS (Static Structural, Workbench 15.0) has been explained.

\subsection{Auto-Cad (2007)}

Auto-Cad is a drafting software application which is used across a wide range of industries. Herein it was used for the accurate 2D design of a scaled model for the estimation of various hydrodynamic forces. The way how the vehicle was modelled is defined in the following steps and further shown in Figure 5:

- Open Auto-Cad and select the desired units from the format option in toolbar.

- Select im command for using and modifying external references in it.

- To generate the outlines of the complex drawing, use spline command for sketching purpose.

- Select valid points to join the original source object into a single object (chassis only).

- Sketch a base line from the bottom of the vehicle to ensure proper ground clearance of the vehicle.

- Scale the selected object based on the reference length and specify a new length by using $s c$ command. The scale factor multiplies the dimensions of the selected objects by the specified scale. A scale factor greater than 1 , enlarges the object, whereas between 0 and 1 , it shrinks the object.

- Save the file as $d x f / d w g$ format.

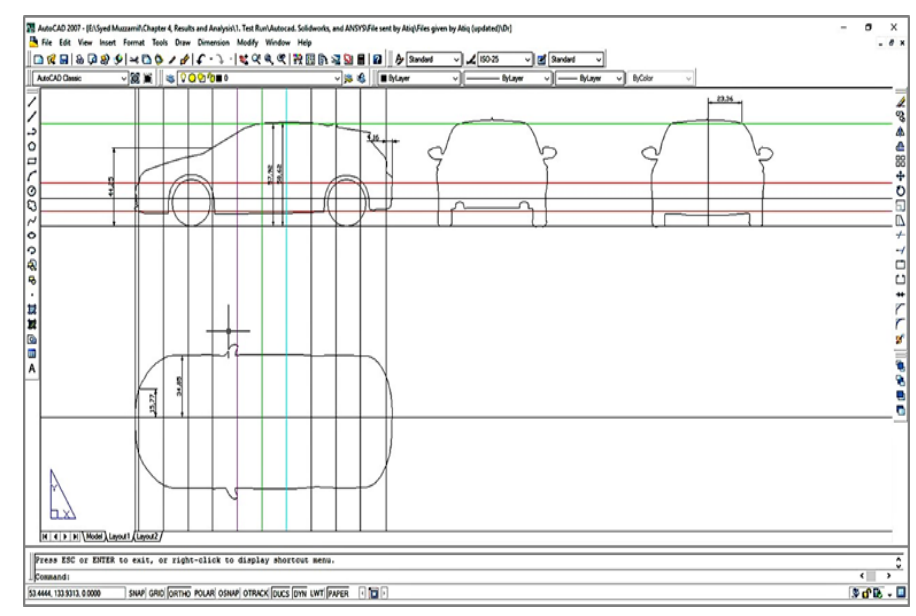

Fig. 5 - 2D model, Autocad

\subsection{Solid-Works (2015)}

Solid-Works is a solid modeler and utilizes a parametric feature-based approach. In the current study, this modeler was used for two main purposes, namely (i) to extrude a 2D model, designed on Auto-Cad into a 3D model by means of extrusion and (ii) to crop the vehicle chassis at the desired floodwater level. The way how it was done is defined in the following steps which is further illustrated in Figure 6:

- The imported part files can contain features or geometry only. Thus, to import a $d x f / d w g$ file as a $2 \mathrm{D}$ sketch: Click open (standard toolbar) or file > open. In the open dialog box, set files of type to $d x f / d w g$, browse to select the file and click open.

- From features toolbar (provides tools for creating model features), select extruded boss/base command. This feature allows to take a 2D sketch and add thickness to it in the third dimension.

- Once the object is selected (vehicle chassis), the boss-extrude toolbar appears. Select blind in direction 1 to specify the end condition which causes extrusion to stop.

- Set the distance to the required width in $d l$ and click $\checkmark$.

- Next, select the extrude cut command which is in command manager. This tool helps to remove sketch profile from a 3D model.

- Once the reference plane is selected, the cut extrude property manager appears. Herein the offset option was selected and the height of extrude cut was set to the desired floodwater depth. Other options available are vertex and surface/face/plane.

- Next, hide the base line from extrude thin option and the remaining object would only be the vehicle chassis.

- Save this file as iges format. 

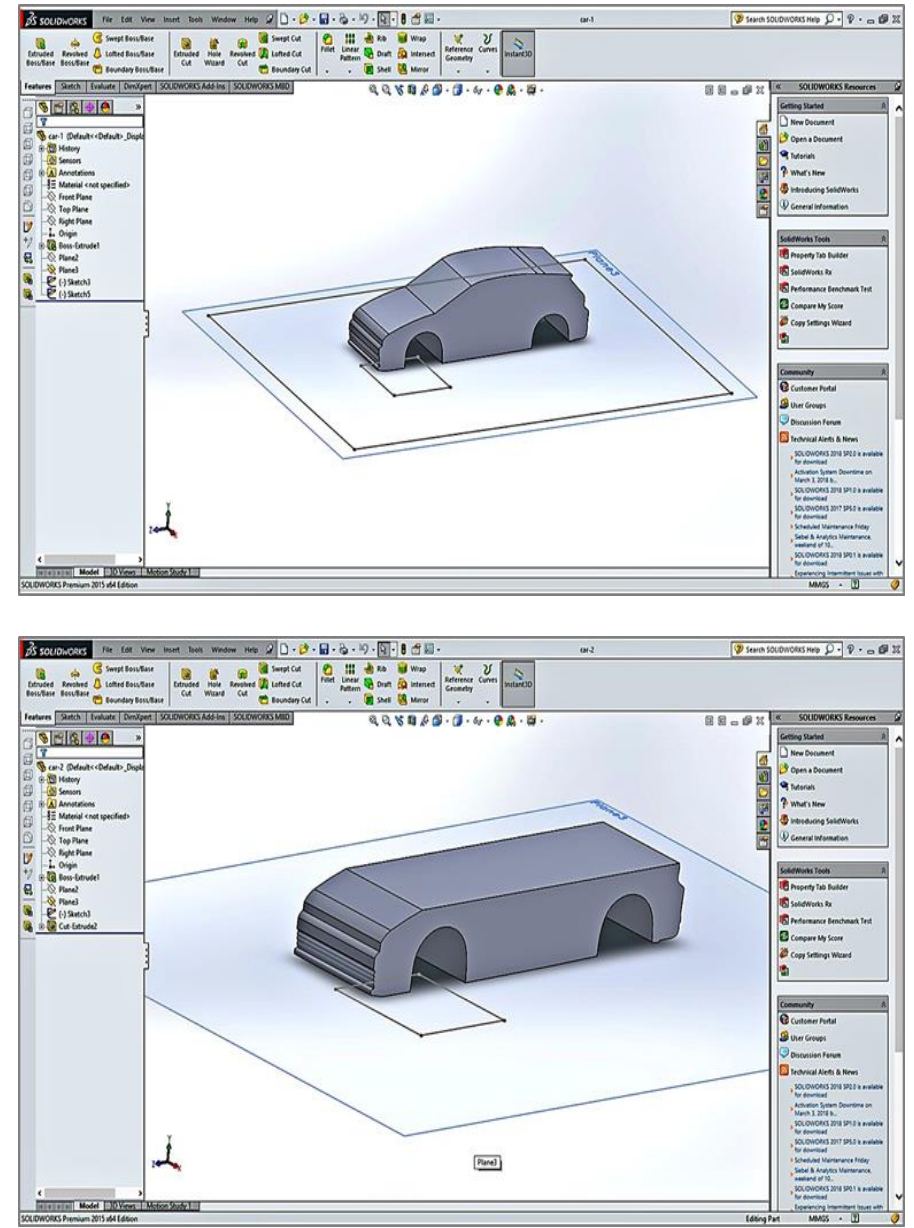

Fig. 6 - Extruded 3D model, Solid-Works

\subsection{ANSYS (Static Structural, Workbench 15.0)}

ANSYS is the global leader in engineering simulations. It develops and markets finite element analysis. However, in the current study it was not used for any simulation purpose as the main concern was to estimate the immersed volume of vehicle at different depths. The way how the submerged volume of the vehicle was estimated is defined in the following steps which is further illustrated in Figure 7:

- Open ANSYS and select static structural from Toolbox.

- Browse and import iges format file and run the model.

- Once the model appears, select properties and the volume of the chassis will appear.

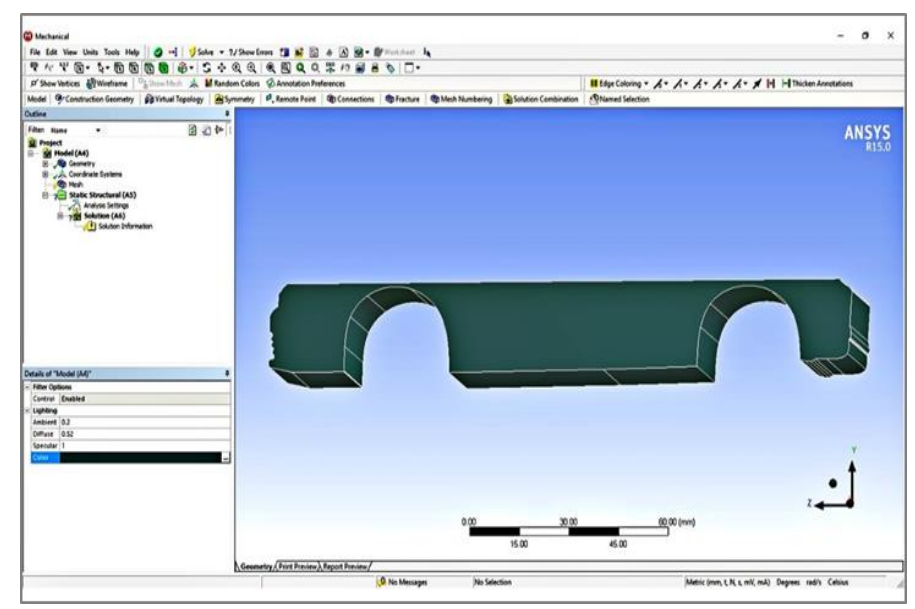

Fig. 7 - Submerged chassis volume, ANSYS 


\section{Results and Discussions}

Under this section, the hydrodynamic forces attained through numerical approach have been conversed. Concerning vertical pushing force, the submerged volume of the vehicle $(V)$ at different water depths was attained. This helped to estimate the buoyancy force $\left(F_{B}\right)$, which further assisted to determine the mode of floating instability. Recall that the impact of lift force $\left(F_{L}\right)$ has been disregarded as it was found insignificant. This happened due to sub-critical state of the flow. Therefore, its impact to cause floating instability was not taken into consideration. On the other hand, to estimate the drag force $\left(F_{D}\right)$, the submerged volume of the vehicle attained at different water depths was fractionated by the vehicle width to obtain submerged area $\left(A_{D}\right)$. Lastly, for the estimation of frictional force $\left(F_{R}\right)$, the friction coefficient $(\mu)$ was taken as 0.3 , whereas the normal reaction force from the ground $\left(F_{N}\right)$, was estimated by deducting the buoyancy force from the vehicle weight (dry conditions).

Based on the numerical results, the study outcomes agreed well with the experimental investigations. The instability thresholds (numerical results) obtained for the static vehicle under the influence of varying hydrodynamic forces are shown in Figure 8. In terms of floating instability, the numerical results complied well with the experimental outcomes, saying that, above the critical water depth, i.e., $0.042 \mathrm{~m}$, the vertical pushing force, namely buoyancy force was found dominant. On the other hand, below this level, the vehicle was found to be stable at limited (v·y) combinations rather than sliding. However, the percentage of error between the experimental investigations and the numerical results was found to be less than $8 \%$. Thus, it can be said that the proposed simple numerical framework has led to the estimation of hydrodynamic impacts on flooded vehicles in an advanced way. Therefore, it is believed that this approach is applicable for the estimation of submerged fractions of static vehicles, i.e., $A_{D}$ and $V$, having complex geometrical design.

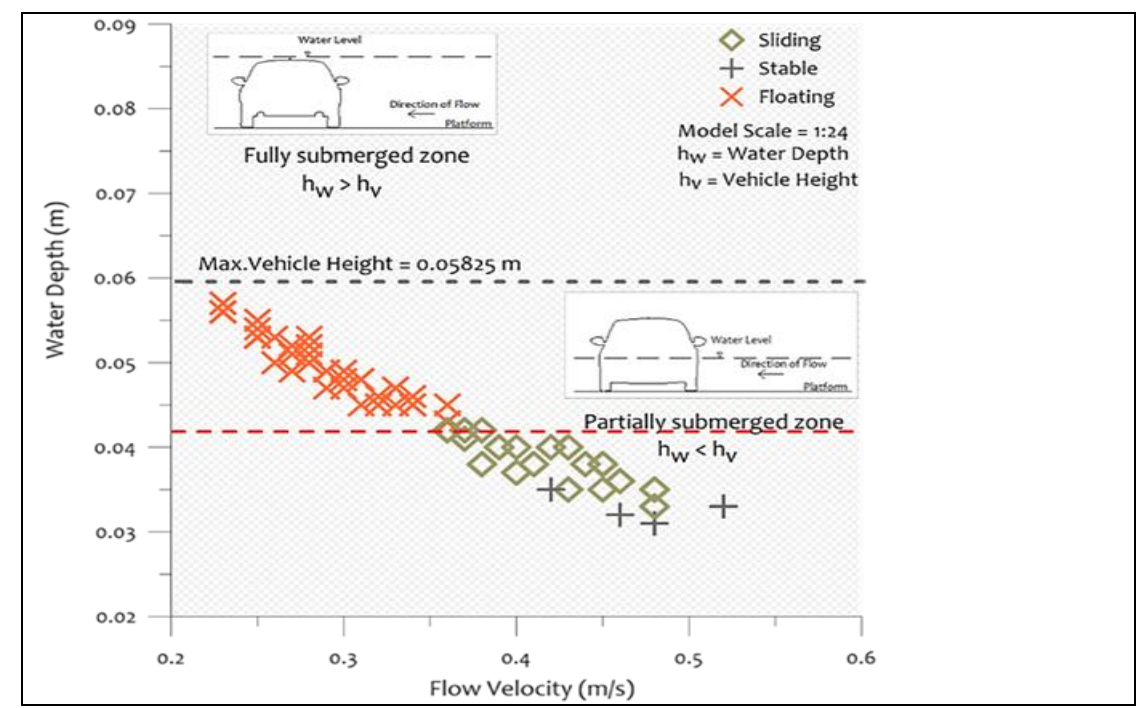

Fig. 8 - Instability threshold values-theoretical analysis

\section{Validations}

Investigations on the hydrodynamics of floodplains with static vehicle was undertaken by conducting a series of experimental studies in the hydraulic flume as well as by determining the impact of hydrodynamic forces on the vehicle by means of numerical approach. Herein the validation of existing research is made through comparison by compiling all the studies carried out on modern static vehicles till date (2010-2017). With that regards, all the studies performed under the similar flow characteristics and submergence level have been compiled. It has been noticed that investigations on several large-scale and small-scale model vehicles, namely 1:14, 1:18, 1:24 and 1:43 have been carried out to understand the criterion of vehicle instability in floodwaters (parked in a car lot), however, those studies were limited to few orientations. Therefore, in the current study, the instability thresholds for the static model vehicle at all orientations, namely $0^{\circ}, 45^{\circ}, 90^{\circ}, 135^{\circ}, 180^{\circ}, 225^{\circ}, 270^{\circ}, 315^{\circ}$ and $360^{\circ}$ towards the direction of incoming flow were obtained. The outcomes of the current research agreed well with the previous investigations with an indication that at high water depths, low flow velocities, whereas at low water depths, high flow velocities were required to cause vehicle instability (partial submergence only) [3], [8]-[10], [12], [14]. The outcomes of the current research together with the previous studies till date are shown in Figure 9. 


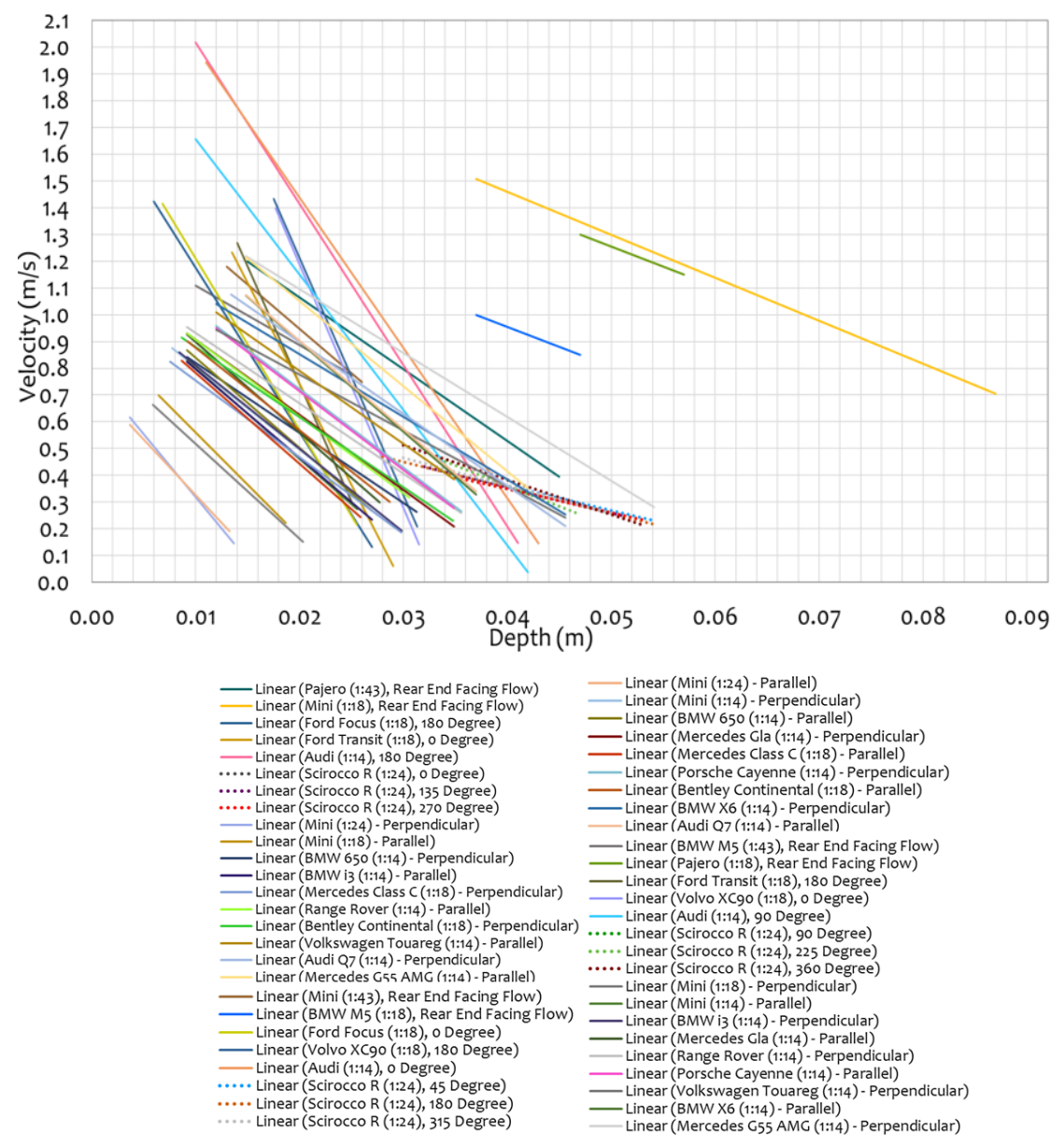

Fig. 9 - Compilation of previous works and validation of current research

\section{Conclusions}

The main findings of the study have highlighted that if the weight, ground clearance, geometric dimensions (length, breadth and width) of the vehicle are known then the proposed numerical framework can be used to estimate the impact of different hydrodynamic forces for the partially submerged vehicles under sub-critical flow conditions. However, pertaining to the limits of floodwater depths and flow velocities to be considered for the estimation of such forces, it can be obtained from the given range of $(\mathrm{v} \cdot \mathrm{y})$ values mentioned in the compilation of previous and existing works. To the author's knowledge, evaluation of coefficients, namely $C_{D}$ and $C_{L}$ have not been quantified well both for full and partial submerged conditions, thus, there is a need for a reliable numerical estimation of such coefficients. It is further recommended that the assessment of flooded vehicles (prototype) under more complex situation needs to be performed in future.

\section{Acknowledgement}

This study was conducted at Universiti Teknologi PETRONAS, Malaysia. This study was undertaken at Universiti Teknologi PETRONAS, Malaysia. This research was supported by Universiti Teknologi PETRONAS (UTP) Internal Grant (URIF 0153AA-G24).

\section{References}

[1] Parker, D.J. (2000). Floods - volume I. Routledge Hazards and Disaster Series, Taylor and Francis Group.

[2] Smith, G. (2015). Expert opinion: Stability of people, vehicles and buildings in flood water. Technical Report 2015/11, Water Research Laboratory, University of New South Wales, Australia.

[3] Martínez-Gomariz, E., Gómez, M., Russo, B., and Djordjević, S. (2017). A new experiments-based methodology to define the stability threshold for any vehicle exposed to flooding. Urban Water Journal, 14(9), 930-939.

[4] Arrighi, C., Castelli, F., and Oumeraci, H. (2016). Effects of flow orientation on the onset of motion of flooded vehicles. Proceedings of the 4th IAHR Europe Congress (Liege, Belgium), 27-29. 
[5] Smith, G. P., Modra, B. D., Tucker, T. A., and Cox, R. J. (2017). Vehicle stability testing for flood flows. Technical Report 2017/07, Water Research Laboratory, University of New South Wales, Australia.

[6] Shand, T., Cox, R., Blacka, M., and Smith, G. (2010). Appropriate safety criteria for vehicles. Project 10: Australian Rainfall and Runoff (AR\&R), Report No. P10/S2/020, Water Research Laboratory, University of New South Wales, Australia.

[7] Wright, K., Doody, B. J., Becker, J., and McClure, J. (2010). Pedestrian and motorist flood safety study: A review of behaviours in and around floodwater and strategies to enhance appropriate behavior. GNS Science Report 2010/51, Institute of Geological and Nuclear Sciences Limited.

[8] Teo, F. Y., Xia, J., Falconer, R. A., and Lin, B. (2012). Experimental studies on the interaction between vehicles and floodplain flows. International Journal of River Basin Management, 10, 149-160.

[9] Shu, C., Xia, J., Falconer, R. A., and Lin, B. (2011). Incipient velocity for partially submerged vehicles in floodwaters. Journal of Hydraulic Research, 49, 709-717.

[10] Teo, F. Y., Falconer, R. A., Lin, B., and Xia, J. (2012). Investigations of hazard risks relating to vehicles moving in flood. Journal of Water Resources Management, 1, 52-66.

[11] Shah, S. M. H., Mustaffa, Z., and Yusof, K. W. (2018). Experimental studies on the threshold of vehicle instability in floodwaters. Jurnal Teknologi, 80(5), 25-36.

[12] Xia, J., Teo, F. Y., Lin, B., and Falconer, R. A. (2011). Formula of incipient velocity for flooded vehicles. Natural Hazards, 58, 1-14.

[13] Bonham A. J., and Hattersley, R. T. (1967). Low level causeways. Report No. 100, Water Research Laboratory, University of New South Wales, Sydney, Australia.

[14] Falconer, R. A., Lin, B., and Xia, J. (2012). 2D hydrodynamic modelling: Mobile beds, vehicle stability and severn estuary barrage. Flood Risk Management Research Consortium. from https://floodrisk.org.uk 